\title{
High-power passively mode-locked tapered InAs/GaAs quantum-dot lasers
}

\author{
D. I. Nikitichev', Y. Ding', M. Ruiz', M. Calligaro ${ }^{2}$, N. Michel$^{2}$, \\ M. Krakowski ${ }^{2}$, I. Krestnikov ${ }^{3}$, D. Livshits ${ }^{3}$, M. A. Cataluna ${ }^{1}$ and E. U. Rafailov' ${ }^{1}$ \\ 1 University of Dundee, School of Engineering, Physics and Mathematics, Dundee, DD1 4HN, UK \\ ${ }^{2}$ Alcatel Thales III-V Lab, 1 Av Augustin Fresnel, Campus de Polytechnique, 91767 Palaiseau, France \\ ${ }^{3}$ Innolume GmbH, Konrad-Adenauer-Allee 11, 44263 Dortmund, Germany \\ Tel: +44 (01382) 386571 \\ Fax: $+44(01382) 388313$ \\ e-mail: d.nikitichev@dundee.ac.uk
}

\begin{abstract}
We report picosecond pulse generation with high peak power in the range of $3.6 \mathrm{~W}$ from monolithic passively mode-locked tapered quantum-dot laser diodes, exhibiting low divergence and good beam quality. These results were achieved using a gain-guided tapered laser geometry. The generation of picosecond pulses with high average power up to $209 \mathrm{~mW}$ directly from such tapered lasers is also demonstrated, corresponding to $14.2 \mathrm{pJ}$ pulse energy (14.65 GHz repetition rate). A comparison between the mode-locking performance of these tapered lasers incorporating either 5 or 10 layers of InAs/GaAs self-organized quantumdots in their active layer is also presented.
\end{abstract}

\section{Introduction}

Semiconductor lasers with high output power and good beam quality are desirable for many commercial and scientific applications, such as medical treatments, biomedical imaging, free-space optical communications, optical pumping of solid-state lasers, laser display technology, material processing, and nonlinear optics [1-7]. Edge-emitting mode-locked semiconductor lasers with flared (tapered) waveguides are well-known for their capability to deliver high output power as well as ultrashort pulses. Tapered lasers typically consist of a straight ridge-waveguide section coupled to a tapered section [2-5]. While the straight waveguide acts as a spatial filter in the cavity, the tapered section of increasing width delivers high power. As a result, tapered lasers show a great potential for providing single spatial mode, good quality beams with high power, as demonstrated by Mar et al. [8]. In this paper, mode locking was achieved in an external cavity configuration, while using a quantum well $\ln _{0.2} \mathrm{Gaa}_{0.8} \mathrm{As}$ tapered laser resulting in the generation of 3.3-ps pulses with $2-\mathrm{W}$ peak power in the waveband of $980 \mathrm{~nm}$ [8]. Alternative technology based on high power passively mode-locked slab-coupled optical waveguide 
lasers has been demonstrated recently [9], achieving average power levels of up to $212 \mathrm{~mW}$, with pulse duration of $25 \mathrm{ps}$ and at a $4.6 \mathrm{GHz}$ repetition rate, resulting in $1.84-\mathrm{W}$ peak power at $1.55-\mu \mathrm{m}$ wavelength, with InGaAsP multi-quantum wells grown on $\ln P$ substrate. Passively mode-locked quantum-dot (QD) lasers have drawn attention of late as efficient compact ultrafast and high-power light sources [10,11]. Mode-locked index-guided tapered lasers based on InGaAs QDs have been demonstrated, generating transform-limited ultrashort pulses with pulse duration of $380 \mathrm{fs}$, average power of $15.6 \mathrm{~mW}$ and peak power of $2.25 \mathrm{~W}$ (at $12{ }^{\circ} \mathrm{C}$ ) [12], although the mechanisms for the generation of transform-limited pulses from such a device without any additional dispersion compensation are not fully understood. In this paper we present the highest peak power (3.6 W) ever achieved from a monolithic passively mode-locked quantum-dot tapered laser by using a gainguided tapered laser geometry.

The fabricated and investigated tapered lasers incorporated either 5 or 10 layers of $\ln G a A s / G a A s$ quantum dots. Picosecond pulse generation with a high average power of $209 \mathrm{~mW}$ corresponding to 14.2 $\mathrm{pJ}$ pulse energy with $14.65 \mathrm{GHz}$ repetition rate is demonstrated, which is more than one order of magnitude higher than previous results [12].

\section{Devices}

The QD epitaxial structures used in the tapered lasers were grown on a GaAs substrate using Molecular Beam Epitaxy (MBE). Two different structures were used, where the active region consisted of either 5 or 10 identical layers of InAs quantum dots separated by $33 \mathrm{~nm}$ GaAs barriers and incorporated in a AlGaAs waveguide with $35 \%$ Al content. The tapered lasers were fabricated with planar gain-guided tapered and straight waveguide section defined only by ion implantation, resulting in a gain-guided geometry (Fig.1). The spontaneous emission coupling factor $(\beta)$ in gain-guided lasers is larger than that in lasers with a comparable active layer volume and with a built-in index waveguide [13]. Owing to the large $\beta$, the spectral width of gain-guided lasers is significantly broader compared to lasers with a built-in index waveguide $[13,14]$. Accordingly, broad spectra of gain-guided lasers could afford potential for narrow pulses compared to index-guided lasers provided all of the bandwidth can be engaged coherently. The tapered sections are $2370 \mu \mathrm{m}$ and $2380 \mu \mathrm{m}$ long for the 5 layers and 10 layers devices respectively, with $2^{\circ}$ taper angle, while the single spatial mode straight sections for mode filtering have a $400-\mu \mathrm{m}$ length in both devices. A reverse bias was applied to the straight waveguide section, which acted as a saturable absorber. The total lengths of the devices were therefore $2.77 \mathrm{~mm}(2.78 \mathrm{~mm})$, resulting in a pulse repetition rate of $14.65 \mathrm{GHz}(14.57 \mathrm{GHz})$. The anti-reflection coating $\left(\mathrm{TiO}_{2} / \mathrm{SiO}_{2}\right)$ on the tapered section 
side and the high-reflection coating $\left(\mathrm{Al}_{2} \mathrm{O}_{3} / \mathrm{Si}\right)^{*} 3$ on the ridge side were $3 \%$ and $95 \%$, respectively. The central operating wavelengths are around $1250 \mathrm{~nm}(1260 \mathrm{~nm})$ for the 5 and 10 layers devices respectively. The lasers were mounted on a Peltier cooler and their operating temperatures were stabilized at $20^{\circ} \mathrm{C}$.

\section{Experimental results}

By changing the driving conditions of the tapered and absorber sections (to which forward and reverse bias were applied respectively), stable mode-locking was achieved for a broad range of current and reverse voltage conditions. The light-current characteristics of the tapered lasers with 5 and 10 QD layers were measured at room temperature, and are shown in Figure 2. The highest output power exceeds $890 \mathrm{~mW}$ at $1800 \mathrm{~mA}$, with a threshold current of $179 \mathrm{~mA}$ for 5-layer QD structure under uniform current injection into both gain and absorber sections. The slope efficiency of about $0.6 \mathrm{~W} / \mathrm{A}$ with lasing wavelength of $\sim 1252 \mathrm{~nm}$ can be obtained. On the contrary, the 10-layer QD laser has slightly lower output power (822 $\mathrm{mW}$ at $1830 \mathrm{~mA})$ and larger threshold current $(211 \mathrm{~mA})$ under uniform injection. The range of driving conditions over which stable mode-locking occurs at 4-V reverse bias for the 5-layer quantum dot laser is illustrated in the upper left inset of figure1. The slope efficiency is estimated at $0.55 \mathrm{~W} / \mathrm{A}$ with lasing wavelength locating at $\sim 1260 \mathrm{~nm}$. We would like to point out that the difference in emission wavelength between the 5- and the 10-layer tapered lasers is not due to temperature variations, as the same bias conditions were applied and the same Peltier cooler was used to stabilise the operating temperature of both lasers. The most probable causes for this difference could be the result of a QD structure discrepancy. In fact, as is well known, an increase in QD size can lead to slightly red-shift of the QD transition wavelength, whereas a minute increase of In content in the capping layer of InGaAs may also produce a red-shift of QD transition wavelength.

The occurrence of stable mode-locking was examined as function of the bias conditions - as represented in Figure 3, the 10-layer QD laser showed a wider mode-locking region compared to 5-layer QD laser. For the 10-layer QD laser we also observed a narrow region where an unstable mode-locking regime was observed. In this regime, a combination of fundamental and harmonic mode-locking was observed, as was evidenced in the RF spectra and in the autocorrelation measurements (not shown here). For the 5 QD-layer laser, the pulse duration and average power were measured as function of applied gain current, for a reverse bias of $-4 \mathrm{~V}$, as shown in Figure 4. A maximum high average power of $209 \mathrm{~mW}$ corresponding to $14.2 \mathrm{pJ}$ pulse energy with 6-ps pulse duration is observed at $-4 \mathrm{~V}$ reverse bias and $1 \mathrm{~A}$ current for this tapered laser. 
The spatial beam characteristics of the 5-layer quantum-dot laser were also investigated in great detail. Far field and near field in the slow axis have been measured with a rotating photodiode and a vidicon camera. The gain-guided structure allows obtaining a stable far field with a low divergence. Indeed, under uniform injection (Figure 5), the far field full width at half maximum (FWHM) varies from $1.7^{\circ}$ to $1.1^{\circ}$ when the current increases from $400 \mathrm{~mA}$ up to $800 \mathrm{~mA}$. The corresponding far-field FWHM values at $1 / \mathrm{e}^{2}$ vary from $3.4^{\circ}$ to $2.6^{\circ}$, and the near field ones from $28 \mu \mathrm{m}$ to $65 \mu \mathrm{m}$. Single-lateral mode with a low divergence was therefore achieved even under a high-current injection, with a beam quality parameter $\mathrm{M}^{2}$ below 2 .

Both tapered lasers - with either 5 or 10 layers of quantum dots - have demonstrated high peak power pulse generation. The highest peak power of $3.6 \mathrm{~W}$ is measured for a driving current of $950 \mathrm{~mA}(1044 \mathrm{~mA})$ and $5.1 \mathrm{~V}(4.9 \mathrm{~V})$ reverse bias for $5(10)$ layers lasers respectively. The corresponding autocorrelation, RF and optical spectra are shown in Figure 6 and Figure 7. Gaussian shapes have been assumed to calculate the pulse width. The combination of a pulse duration of $3.2 \mathrm{ps}(3.3 \mathrm{ps})$, and an optical spectrum FWHM of $7.3 \mathrm{~nm}(8.4 \mathrm{~nm})$ results in a time-bandwidth product (TBWP) of $4.4(5.2)$ measured from the 5 and 10-layer devices accordingly. The generated pulse is therefore not transform-limited, which opens the possibility for significant pulse post-compression, which the potential to boost the peak power up to $20 \mathrm{~W}$ by theoretical estimation. Future investigation work could be pursued by fabricating lasers with a smaller gain-to-absorber length ratio, for instance from 6:1 (as in the present work) to 4:1, in order to hopefully generate shorter pulses as described in ref. 12.

\section{Conclusion}

Novel tapered quantum-dot lasers with a gain-guided geometry operating in a passively mode-locked regime have been fabricated and investigated, using structures that incorporated either 5 or 10 layers. The generation of picosecond pulses with high average power of up to $209 \mathrm{~mW}$ was demonstrated, corresponding to $14.2 \mathrm{pJ}$ pulse energy. We demonstrate a low slow axis far-field, which remains stable even under a high-current injection. Furthermore, the highest peak power of $3.6 \mathrm{~W}$ is achieved for a tapered quantum-dot laser (corresponding to a pulse duration of $3.2 \mathrm{ps}$ ), with a time-bandwidth product that offers the potential of further pulse post-compression for boosting the peak power up to 10 times and thus forming the basis of an extremely compact ultrafast laser system. 


\section{Acknowledgement}

The work was funded within the Seventh Framework Program "FAST-DOT", through Grant No. 224338.

M. A. Cataluna acknowledges also financial support through a Royal Academy of Engineering/EPSRC Research Fellowship. The authors would like to acknowledge Yannick Robert for facet treatments and Eric Vinet for laser mounting.

\section{References}

[1] R. Diehl, Ed., High-power diode lasers: fundamentals, technology, applications (Topics in applied physics 78). Berlin, Germany: Spinger-Verlag, 2000.

[2] N. Michel, M. Ruiz, M. Calligaro, Y. Robert, M. Lecomte, O. Parillaud, M. Krakowski, I. Esquivias, H. Odriozola, J. M. G. Tijero, C. H. Kwok, R. V. Penty, I. H. White, Proc. of SPIE, vol. 7616, 76161F-1, 2010.

[3] K. Paschke, B. Sumpf, F. Dittmar, G. Erbert, R. Staske, H. Wenzel, and G. Trankle, IEEE J. Sel.Top. Quantum Electron. 11, 1223 (2005).

[4] B. Sumpf, KH. Hasler, P. Adamiec, F. Bugge, F. Dittmar, J. Fricke, H. Wenzel, M. Zorn, G. Erbert, G. Trankle, IEEE J. Sel.Top. Quantum Electron. 15, 1009 (2009).

[5] M. T. Kelemen, J. Weber, G. Kaufel, G. Bihlmann, R. Moritz, M. Mikulla, and G. Weimann, Electron. Lett. 41, 1011 (2005).

[6] K. Kim, S. Lee and P. J. Delfyett, Optics Express, 13, 4600 (2005).

[7] E. Innerhofer, T. Sudmeyer, F. Brunner, R. Paschotta, U. Keller, Laser Phys. Lett. 1, 82 (2004).

[8] A. Mar, R. Helkey, W.X. Zou, D.B. Young, and J.E. Bowers, Appl. Phys. Lett. 66, 3558 (1995).

[9] F. R. Ahmad and F. Rana, IEEE Photonics Tech. Lett. 20, 190 (2008).

[10] E. U. Rafailov, M. A. Cataluna, W. Sibbett, Nature Photonics 1, 395 (2007).

[11] W. Kaiser, J. P. Reithmaier, A. Forchel, H. Odriozola, and I. Esquivias, Appl. Phys. Lett. 91, 051126 (2007).

[12] M. G. Thompson, A. Rae, M. Xia, R. V. Penty and I.H. White, IEEE J. Sel.Top. Quantum Electron. 15, 661 (2009).

[13] K. Petermann, IEEE J. Quantum Electron. 15, 566 (1979).

[14] G. P. Agrawal, J. Opt. Soc. Am. B, 1, 406 (1984).

\section{Figure Captions}

Figure 1. Schematic of the two-section gain-guided lasers with 5 or 10 quantum dot layers (L1- absorber section, L2- gain section).

Figure 2. Light-current $(L-I)$ characteristics for fully connected 5-layer and 10-layer quantum dot devices. Upper left inset: $L-I$ characteristics obtained for an applied absorber bias of $-4 \mathrm{~V}$. Lower right inset: dependence between the threshold current and the absorber bias,

Figure 3. Mapping of mode-locking regimes observed a) 5-layer quantum dot laser b) 10 layer quantum dot laser. 
Figure 4. a) Pulse duration and b) average power dynamics at 4-V reverse bias for the 5-layer quantum dot laser.

Figure 5. Far-fields under uniform injection for the 5-layer quantum dot laser.

Figure 6. a) Autocorrelation, b) RF spectrum and c) optical spectrum for an injection current of $950 \mathrm{~mA}$ and reverse bias $5.1 \mathrm{~V}$ for high peak power regime for the 5-layer quantum dot laser.

Figure 7. a) Autocorrelation, b) RF spectrum and c) optical spectrum for an injection current of $1044 \mathrm{~mA}$ and reverse bias $4.9 \mathrm{~V}$ for high peak power regime for the 10 -layer quantum dot laser. 


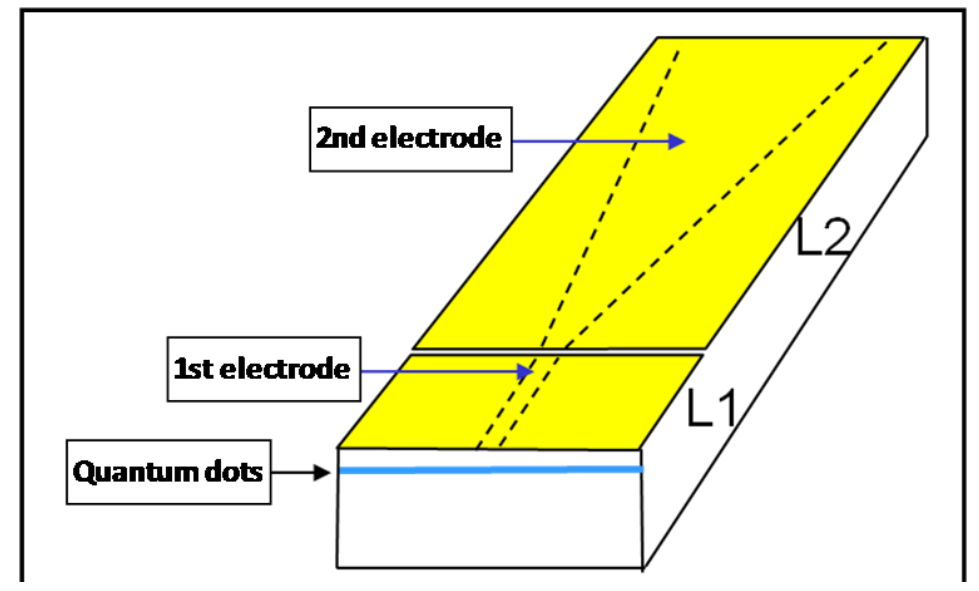

Figure 1. Schematic of the two-section gain-guided lasers with 5 or 10 quantum dot layers (L1- absorber section, L2- gain section). 


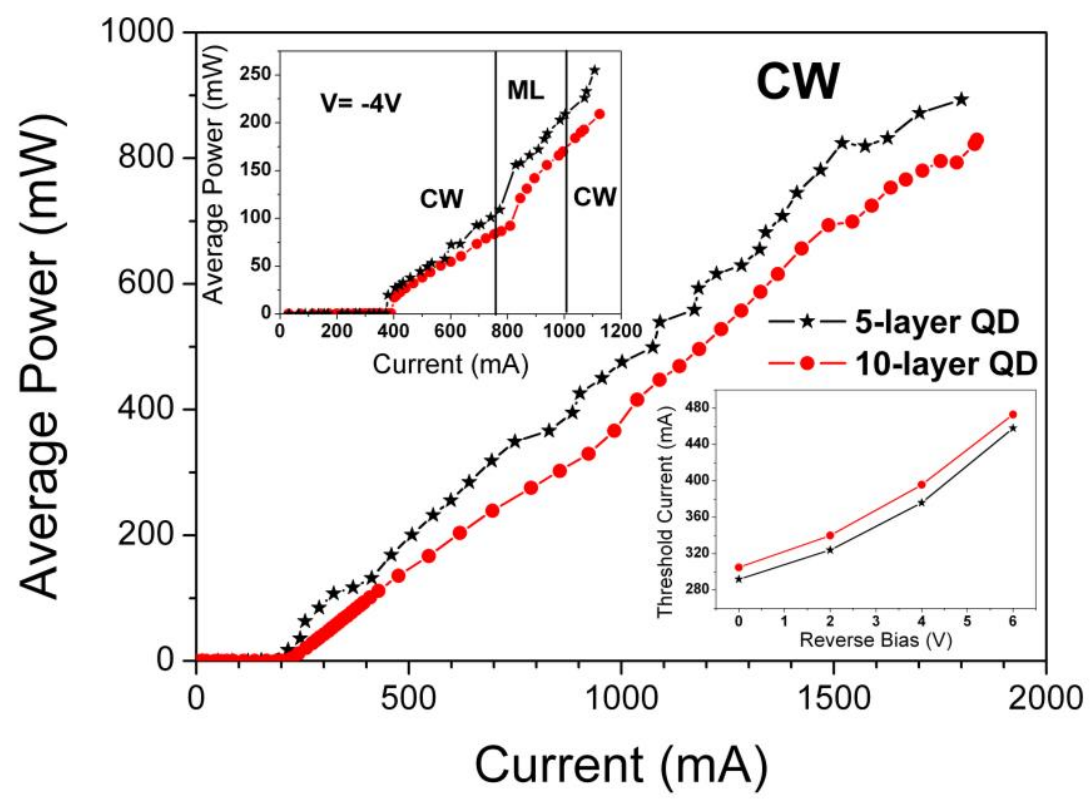

Figure 2. Light-current ( $L-I)$ characteristics for fully connected 5-layer and 10-layer quantum dot devices. Upper left inset: $L-I$ characteristics obtained for an applied absorber bias of $-4 \mathrm{~V}$. Lower right inset: dependence between the threshold current and the absorber bias. 

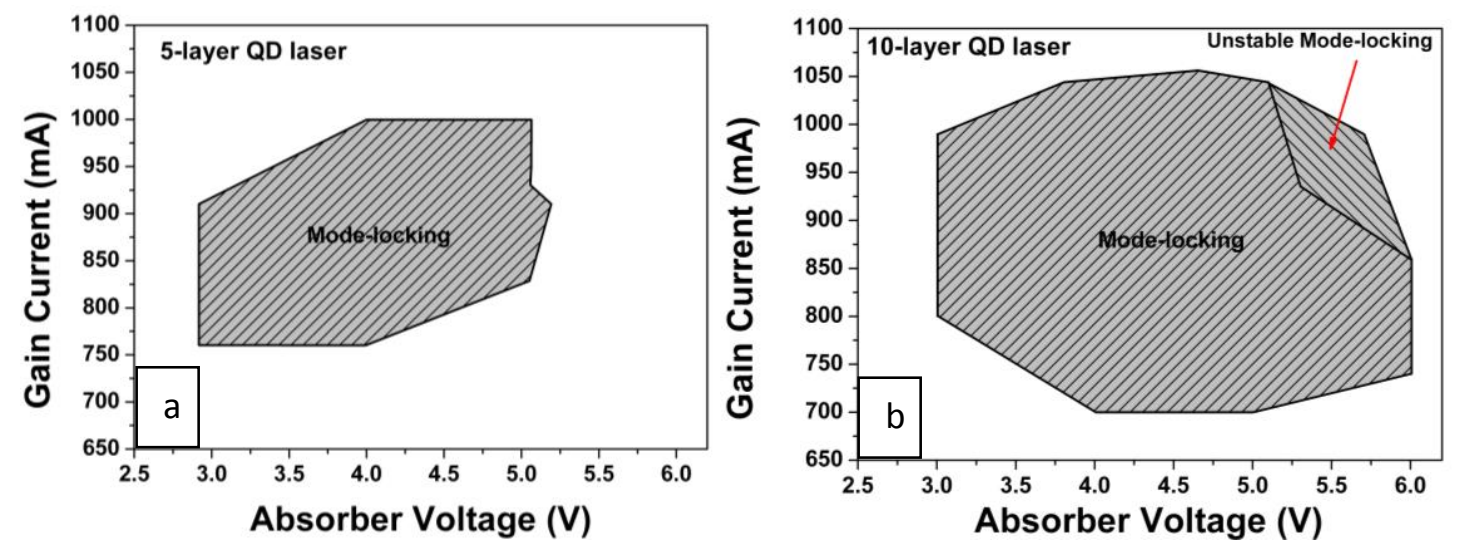

Figure 3. Mapping of mode-locking regimes observed a) 5-layer quantum dot laser b) 10 layer quantum dot laser. 

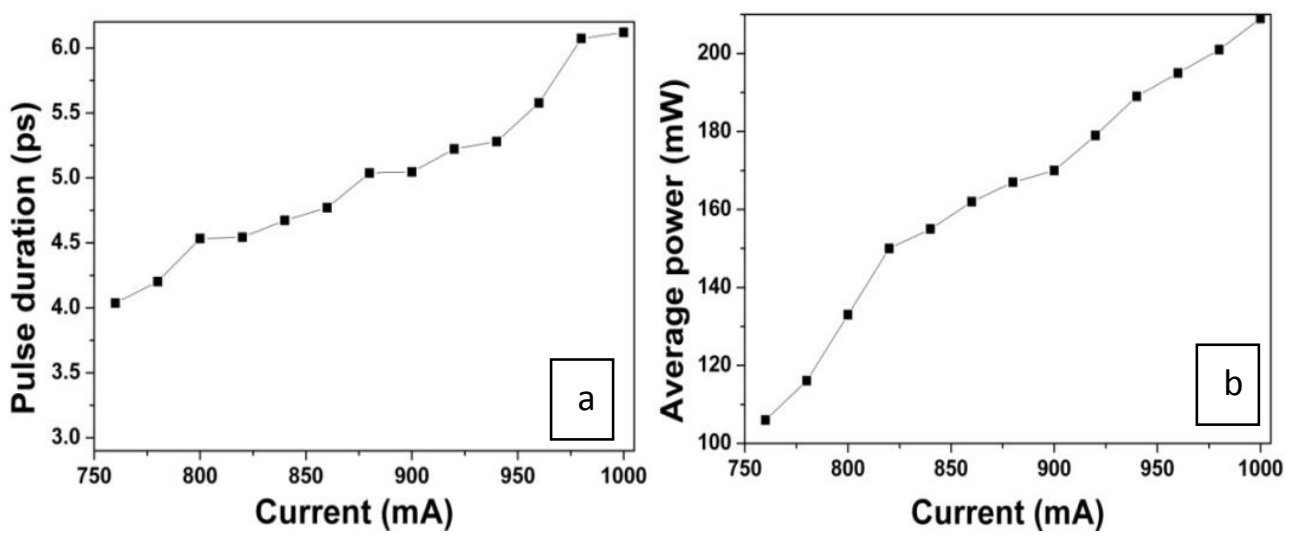

Figure 4. a) Pulse duration and b) average power dynamics at 4-V reverse bias for the 5-layer quantum dot laser. 


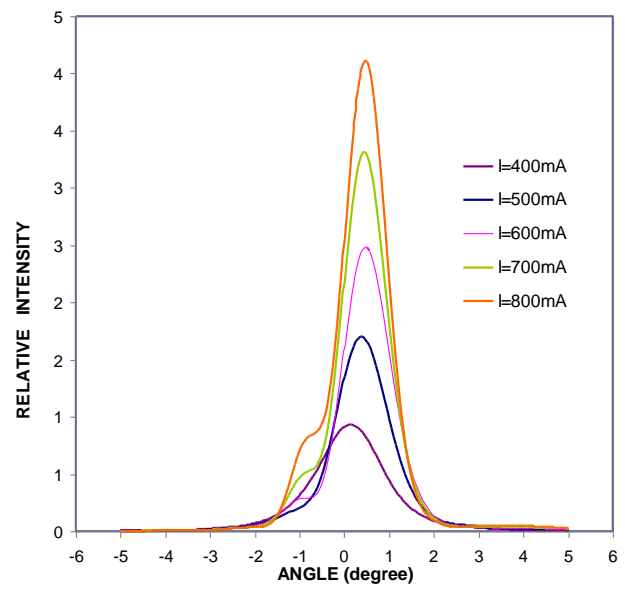

Figure 5. Far-fields under uniform injection for the 5-layer quantum dot laser. 

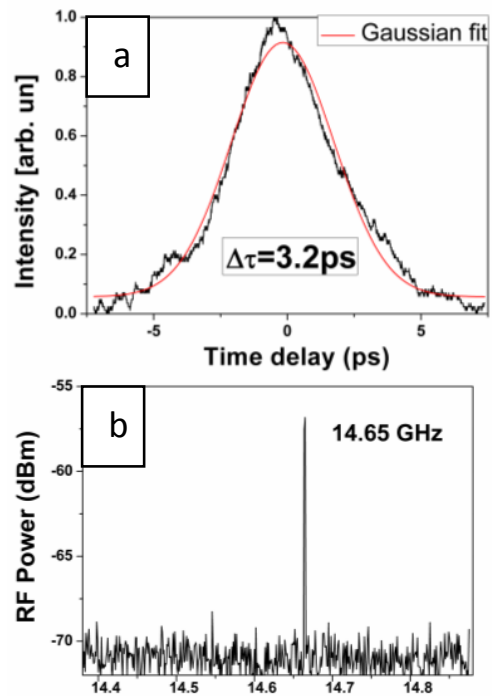

Frequency $(\mathrm{GHz})$

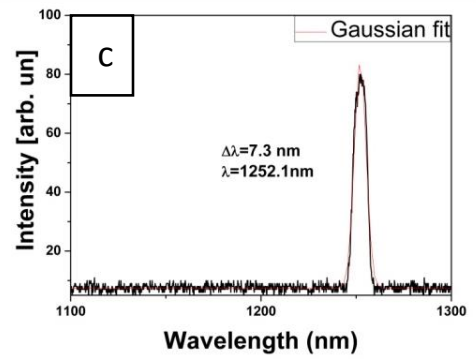

Figure 6. a) Autocorrelation, b) RF spectrum and c) optical spectrum for an injection current of $950 \mathrm{~mA}$ and reverse bias $5.1 \mathrm{~V}$ for high peak power regime for the 5-layer quantum dot laser. 

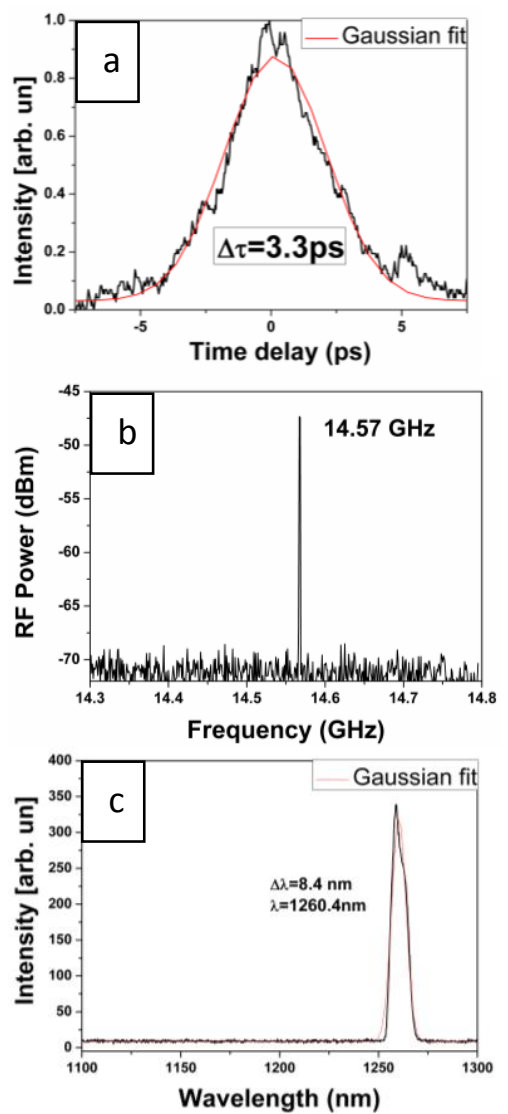

Figure 7. a) Autocorrelation, b) RF spectrum and c) optical spectrum for an injection current of $1044 \mathrm{~mA}$ and reverse bias $4.9 \mathrm{~V}$ for high peak power regime for the 10-layer quantum dot laser 